\title{
Keyhole coherent diffractive imaging
}

\author{
BRIAN ABBEY1 ${ }^{1}$ KEITH A. NUGENT¹ ${ }^{1}$ GARTH J. WILLIAMS ${ }^{\star *}$, JESSE N. CLARK², ANDREW G. PEELE², \\ MARK A. PFEIFER ${ }^{2}$, MARTIN DE JONGE ${ }^{3}$ AND IAN MCNULTY ${ }^{3}$ \\ ${ }^{1}$ School of Physics, The University of Melbourne, Victoria 3010, Australia \\ ${ }^{2}$ Department of Physics, La Trobe University, Bundoora, Victoria 3086, Australia \\ ${ }^{3}$ Advanced Photon Source, Argonne National Laboratory, Argonne, Illinois 60439, USA \\ *e-mail: garthw@unimelb.edu.au
}

The availability of third-generation synchrotrons and ultimately $\mathrm{X}$-ray free-electron lasers ${ }^{1}$ is driving the development of many new methods of microscopy. Among these techniques, coherent diffractive imaging (CDI) is one of the most promising, offering nanometre-scale imaging of non-crystallographic samples. Image reconstruction from a single diffraction pattern has hitherto been possible only for small, isolated samples, presenting a fundamental limitation on the CDI method. Here we report on a form of imaging we term 'keyhole' CDI, which can reconstruct objects of arbitrary size. We demonstrate the technique using visible light and $\mathrm{X}$-rays, with the latter producing images of part of an extended object with a detectorlimited resolution of better than $20 \mathrm{~nm}$. Combining the improved resolution of modern $\mathrm{X}$-ray optics with the wavelength-limited resolution of CDI, the method paves the way for detailed imaging of a single quantum dot or of a small virus within a complex host environment.

In 1982, it was shown that it is possible to use the information contained in a far-field diffraction pattern to 'almost uniquely' reconstruct an isolated object ${ }^{2}$. Numerous experimental demonstrations for simple objects followed ${ }^{3-5}$. So far, the technique has been used to investigate a variety of complex problems, from tomographic mapping of strain fields inside a nanocrystal ${ }^{6}$ to high-resolution imaging of a yeast cell ${ }^{7}$. Phase information from a diffracted wavefield is not explicitly recorded in the measured intensity and must be extracted from the diffraction pattern; all coherent diffractive imaging (CDI) techniques therefore involve some form of phase retrieval. To enable the phase determination, the intensity distribution must be sampled at a spacing that is finer than its Nyquist frequency ${ }^{8}$, which in turn requires that the sample be finite. For the case of an object with a boundary inside the incident wavefield, the sampling criterion can often be satisfied so that, for all practical purposes, a unique solution exists.

With a finite, diverging beam, it is possible to illuminate a small isolated region within a large object. In this letter we show that the illumination itself can be used to define the boundary of an effective object giving rise to a finite wave exiting the sample. We discuss our results in terms of the exit surface wave (ESW), which we define as the modification to the incident wave caused by interaction with the sample. The sample size requirements on the imaged object in plane-wave CDI here apply to the spatial extent of the ESW; no limitations are therefore placed on the spatial extent of the object itself.

In keyhole coherent diffractive imaging (KCDI), a beam is focused and the object is placed downstream of the focal point so that it is illuminated by a diverging wave ${ }^{9}$. Thus, although the measurement takes place in the far field, the diffracted wave is related to the object via the Fresnel diffraction formalism so that, for an ESW of finite extent, a unique solution for the phase of the diffraction pattern is guaranteed ${ }^{10}$. Image reconstruction generally involves iteratively determining a complex wave that is consistent with the measured intensity ('modulus' constraint) and some known experimental information, such as the size and/or shape of the ESW ('support' constraint). Here, we iterate using the error reduction ${ }^{11}$ (ER) algorithm to solve for the phase of the diffracted wave in the plane of the detector. It has been shown that this method finds a unique solution rapidly and reliably in the Fresnel regimes.

The experimental set-up for the optical and X-ray experiments is conceptually identical and is shown schematically in Fig. 1. The experimental geometry is similar to that proposed in ref. 12 in 1949 for in-line holography; however, the requirements on the source and detector are substantially different. Further details of the experiments and reconstruction algorithms are given in the Methods section. The test object for the optical experiments was a USAF 1951 resolution target. The measured data consist of two regions: the central region, in which the unscattered beam interferes with the scattered light, and the high-angle scatter region, where diffracted intensity is measured beyond the numerical aperture of the focusing lens (see Fig. 1, inset).

A movie of the sample being scanned through the focal plane, in which a sample image is recovered independently in each frame, can be viewed in a Supplementary Information video. Image reconstructions at two positions from the lens, $1.06 f$ and $1.27 f$ (where $f$ is the focal length), with the beam incident on the same region of the extended sample, are shown in Fig. 1. The spatial resolution of the image is essentially identical for both fields of view and determined by the numerical aperture of the detector, which we define as the half-angle subtended by the detector at the sample plane.

Figure 2 shows the central portion of the resolution target and corresponding CDI reconstruction. By collecting data from different areas on the sample, any region of an extended object can be imaged. The concept is demonstrated in Fig. 2b, in which four images of the sample have been combined after independent reconstruction. Using Abbe theory ${ }^{13}$, the detector numerical aperture gives a predicted spatial resolution of $8 \mu \mathrm{m}$ (see the Methods section). As can be seen from the inset in Fig. 2b, the smallest features that we are able to clearly resolve indicate a resolution that is consistent with this expectation. 


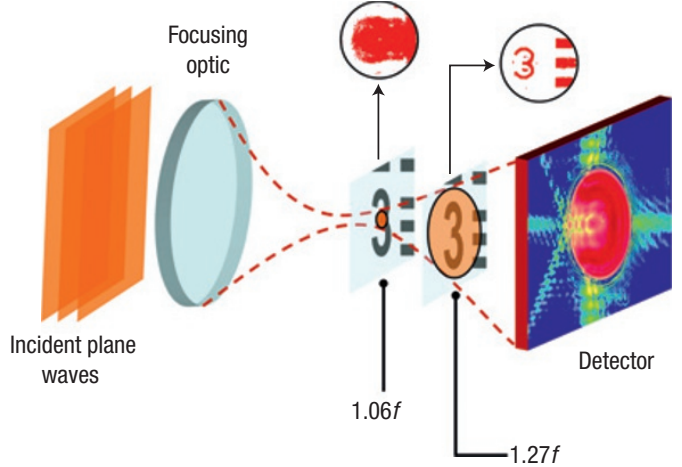

Figure 1 Schematic diagram showing the experimental geometry used for optical and soft-X-ray measurements. Two different positions for the sample relative to the focus are indicated. The arrows point to visible-light reconstructions of the Arabic numeral three; the one furthest from the focus has an illumination spot of $5.4 \mathrm{~mm}$ at the sample and shows the whole numeral plus part of three lines. The other, with an illumination spot of $1.1 \mathrm{~mm}$, is a reconstruction of just the central tine of the numeral. Also shown is a logarithmic plot of the intensity (right) corresponding to the 'high-magnification' reconstruction (left) with the sample close to the focal plane.

The iterative algorithms used to reconstruct an image of the diffracting sample implicitly assume a fully coherent incident wavefield. For the optical (laser) experiments this assumption is justified. Partially coherent light from a third-generation synchrotron, however, can have a detrimental influence on our ability to obtain a reliable reconstruction ${ }^{14}$. Having demonstrated CDI using an illumination-defined support at optical wavelengths, we now report on experiments in the scientifically important $\mathrm{X}$-ray regime.

X-rays $(2.17 \mathrm{keV})$ were used to illuminate part of a spoked, star-shaped, resolution test pattern (Fig. 3a) with a finest feature size of $40 \mathrm{~nm}$. Figure $3 \mathrm{~b}$ shows the coherent X-ray diffraction pattern from this sample, which extends to the edges of the array on all four sides. Figure $3 \mathrm{c}$ shows that we are able to reconstruct the propagated ESW out to the highest angle at which diffracted $\mathrm{X}$-rays could be detected. Careful inspection of both measured and reconstructed wavefield magnitudes reveals excellent agreement between the diffraction pattern produced by the two rings of the star pattern and the reconstructed ESW.

A scanning transmission X-ray micrograph with a step size of $25 \mathrm{~nm}$ is shown in Fig. 3d. The scanned area is $\sim 4.8 \times 5.2 \mu \mathrm{m}$ in size. Although the overall shape of the sample can be easily determined, the fine structure of the inner ring of the star pattern is not resolved. Figure 4 shows the image reconstructed from the diffraction pattern in Fig. 3a, clearly demonstrating that CDI produces a resolution that is significantly better than that of the zone plate.

The diameter of the total field of view of the CDI reconstruction is $\sim 8.5 \mu \mathrm{m}$, which is less than the $9.6 \mu \mathrm{m}$ diameter across the two inner rings of the star pattern. Comparison of the reconstructed image with the technical specifications for the resolution targets shows that the two are consistent. In particular, note that the spokes missing from both the inner ring and outer ring of the star are also clearly missing from the bottom left-hand corner of the reconstruction. Lineouts taken through the inner ring of spokes (top left, Fig. 4) show that the object has sharply defined edges. The phase, which varies with the thickness of the diffracting object, drops to the minimum value at the edge of the spokes within $20 \mathrm{~nm}$. Hence, the experimentally determined resolution is consistent with the expected value of $16 \mathrm{~nm}$ and yields a resolution for CDI that is a
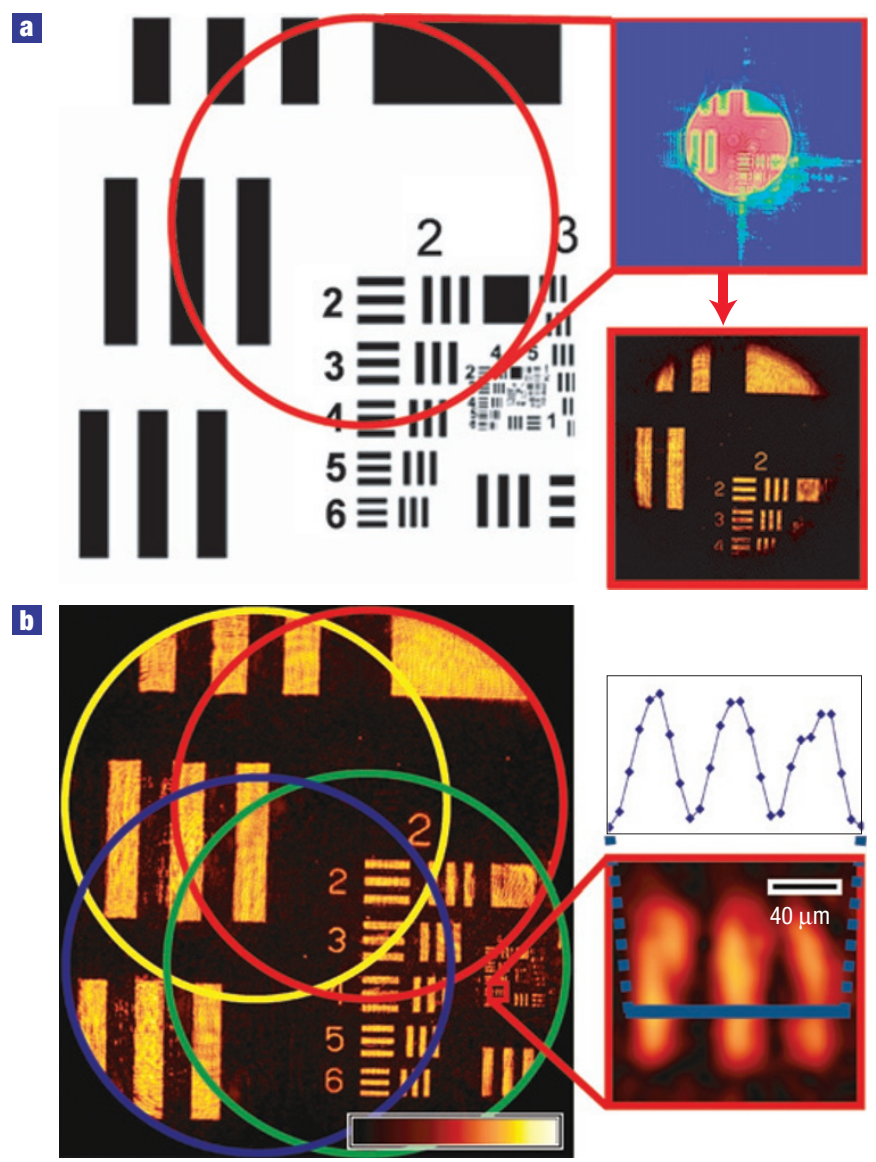

Figure 2 Visible-light data. a, USAF 1951 resolution target. The circle indicates one of four areas that were illuminated with a finite spherical beam diverging from the focus. The reconstruction of the amplitude of the ESW from one of these areas is shown in the corner of the figure. $\mathbf{b}$, A collage of the corresponding four separate reconstructions of the extended object. Inset: Expanded view of the smallest resolvable line pair (group 4, element 5 of USAF 1951 resolution target); a lineout taken through the centre is also shown. Note that the resolution is consistent with the expected value for this experimental geometry.

a factor of three better than that obtained using the same optics in scanning transmission X-ray micrography (STXM) mode (see the Methods section).

The sample here is placed sufficiently close to the focal plane of the zone plate that the illumination contains rapid variations in amplitude, particularly in the region contained within the geometric shadow of the central stop. We attribute the artifact near the centre of the reconstructed image to small inaccuracies in our characterization of the illumination close to the optical axis. We note that this effect is not present in reconstructions of other samples $^{9}$ and that it is not characteristic of the method.

It is important to distinguish the KCDI approach with an illumination-defined region of interest from the ptychography method $^{15}$. The ptychographical algorithm uses the additional information provided by the overlap of many diffraction patterns from adjacent regions to reconstruct an image. The quality of the reconstruction thus obtained depends on the amount of overlap of each dataset and knowledge about the position of the beam on the sample at each location. In KCDI each reconstruction is carried out independently on the basis of the information contained within a single diffracted intensity measurement and knowledge of the 
a

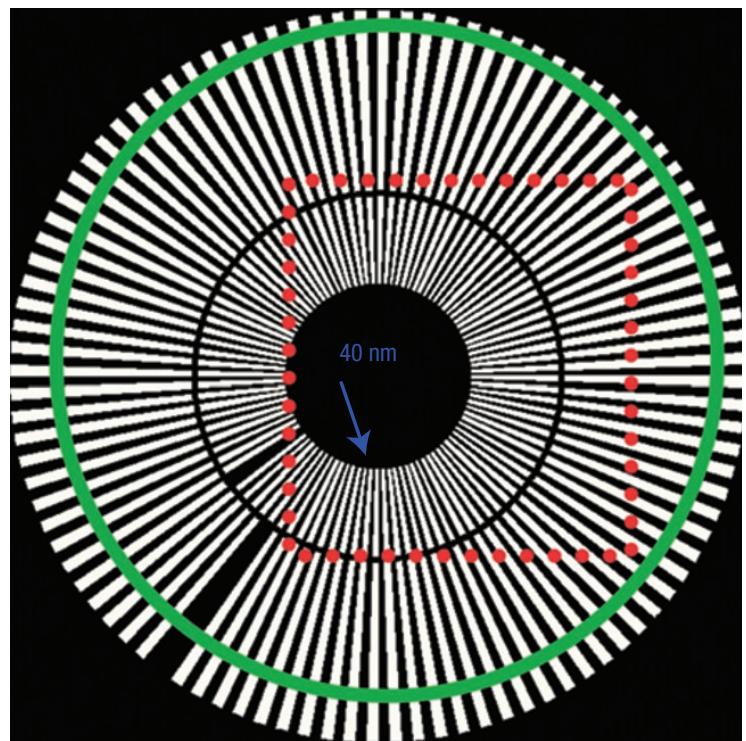

$\mathbf{6}$

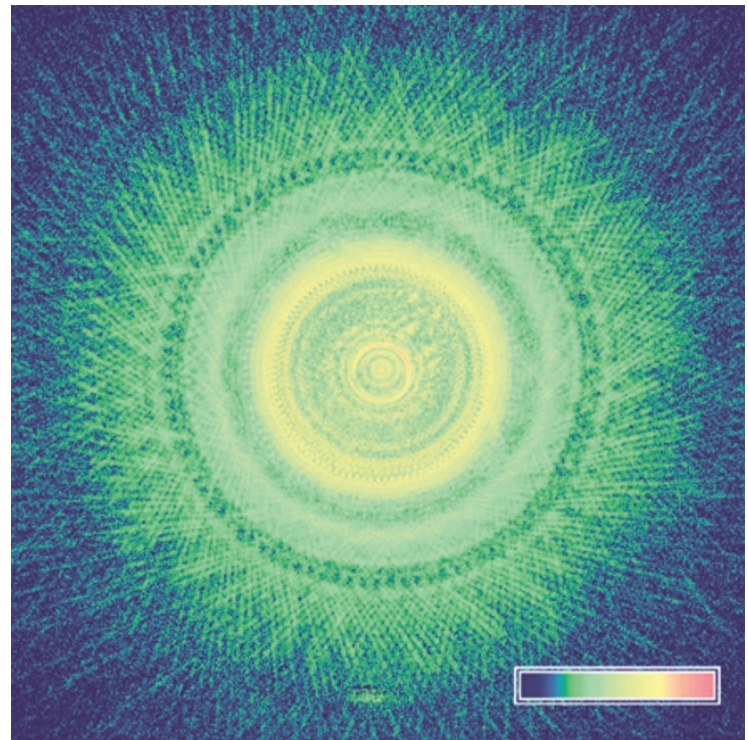

b

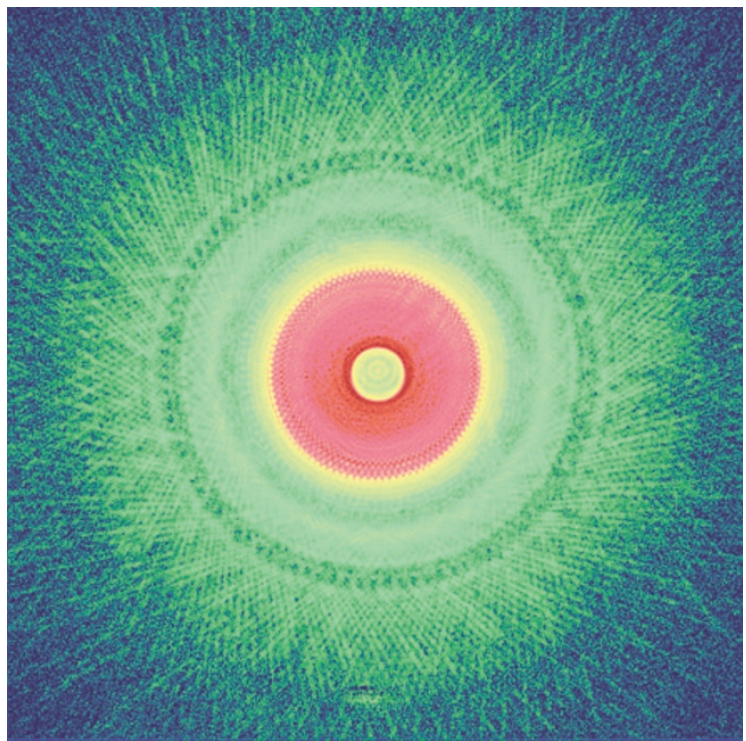

d

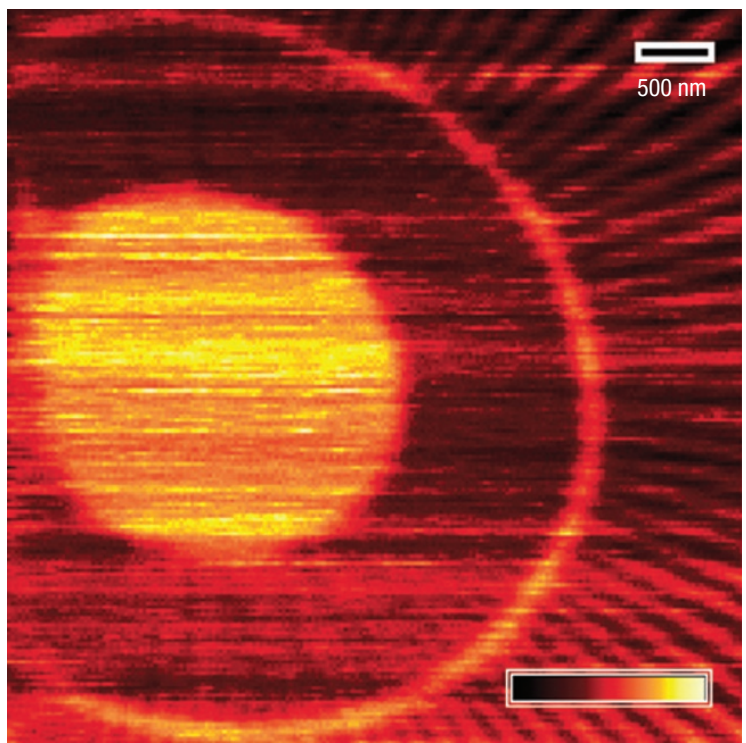

Figure 3 Synchrotron X-ray data. a, A technical drawing for the central region of the spoked star pattern; the region scanned by STXM (red rectangle) and the area illuminated for the KCDI reconstruction (green circle) are indicated. $\mathbf{b}$, The measured intensity $\sqrt{I^{\mathrm{m}}}$ at the detector. c, The reconstructed ESW propagated to the detector plane. The data in $\mathbf{b}$ and $\mathbf{c}$ are logarithmically scaled to show the high-angle scatter. The interference with the incident beam caused by the outer ring of spokes (smallest separation $=80 \mathrm{~nm}$ ) of the star pattern is visible at the edge of the illumination in both $\mathbf{b}$ and $\mathbf{c}$. The inner ring of spokes (refer to a for sample geometry) is also contained within the illumination. In this region, the spoke separation is generally less than the focal spot size of the FZP $(\sim 63 \mathrm{~nm})$ and hence cannot be resolved. Note that ideally $\mathbf{c}$ is equal to $\mathbf{b}$ minus the incident illumination. $\mathbf{d}$, An STXM image of the sample. The horizontal lines are characteristic of STXM imaging and could be either reduced or removed through normalization by the incident intensity. The non-orthogonality in the image is due to errors in the scan stage.

incident wavefield ${ }^{9}$. Thus any area of the sample can be imaged with an appropriate illumination. For both ptychography and KCDI, repeat exposures to image large areas of an extended object could induce sample damage. However, in the present case, the amount of overlap between exposures is arbitrary and may be tailored to the sample.

We note that the measurement described here has the potential to be applied to a range of other wavefields. To use the illumination to define a region of interest it must rapidly fall to an experimentally negligible value at the edges and so must be created by a high-quality, but not necessarily high-resolution, focusing optic.
Further, the quality of the reconstruction depends critically on the knowledge of the illuminating beam, which must be reconstructed with a resolution equal to or greater than that of the ESW. We also know from previous experiments that the coherence of the illumination plays an important role in $\mathrm{CDI}^{14,16}$. The above are all issues that would need to be addressed for imaging using, for example, electrons, but there would seem to be no major impediment to them being successfully solved and for the method to find application in high-resolution electron imaging ${ }^{17}$.

In conclusion, we have demonstrated that the field of view, as defined by the illumination in the plane of the sample, can 


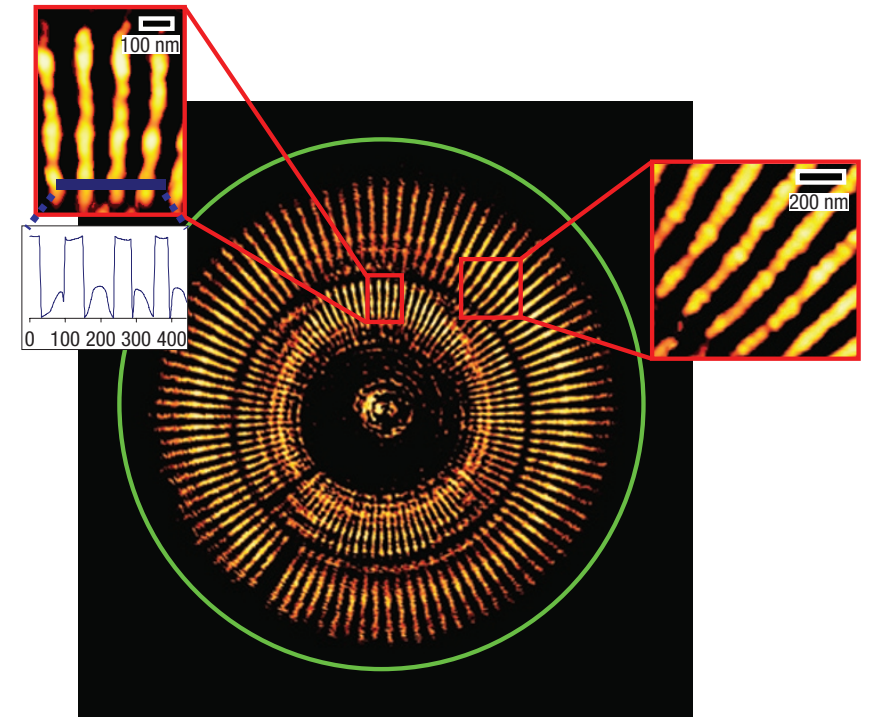

Figure 4 Reconstruction of the ESW represented as the product of its amplitude and phase and thresholded to show only the real component of the complex wave (see the Methods section). Enlargements of portions of the second ring (right) and innermost ring (top left) are also shown. The spokes in the inner ring are well resolved and indicate a spatial resolution of better than $20 \mathrm{~nm}$. The green circle represents the extent of the illumination spot that lies inside the boundary of the star pattern (Fig. 3a). The pattern is reconstructed to the boundary of the circle; it is only the linear scaling of this image that causes it to vanish. Note that because the phase contrast is more pronounced for this sample and photon energy the lineout in the figure was taken through the phase component (without thresholding) of the complex ESW (shown in Supplementary Information, Fig. S1).

be used as the support constraint for CDI with a phase-curved incident illumination, enabling imaging of extended objects from a single measurement of the diffracted intensity. The convergence to a unique solution using the illumination as the support does not introduce any additional ambiguities into the reconstruction and so we retain the benefits of imaging with a curved wavefield, such as rapid convergence without stagnation, whilst being able to image an object that extends well beyond the illuminating beam. We believe that a number of interesting scientific applications of this technique will arise from the fact that the size of the illumination spot in the sample plane may be adjusted by simply changing the defocus position of the sample. This feature is unique to the experimental geometry shown in Fig. 1 and means that a very small, high-intensity illumination spot can be used to select a region of interest on the sample.

This technique will find immediate application in the highresolution imaging of a wide variety of samples previously inaccessible to CDI. In addition, it will enable the study of weakly scattering targets where a large flux density is required for imaging of selected areas and where beam damage must be considered. Hence, by reconstructing small fields of view within extended objects, this form of coherent diffractive imaging will be applicable to many outstanding problems in both the materials and biological sciences.

\section{METHODS}

A USAF 1951 positive test pattern (purchased from Edmund Optical) was used for the optical measurements. The sample (Fig. 2) was positioned $127 \mathrm{~mm}$ from the focus and consisted of chromium (density $>3.0$ ) vacuum-deposited onto a clear glass substrate with a finest feature size of $\sim 2 \mu \mathrm{m}$. A $\sim 0.01 \mathrm{~mW}$ laser beam $(632 \mathrm{~nm})$ passing through a $\times 15$ beam expander was used as the coherent source. The focusing optic was a biconvex spherical lens with a focal length $(f)$ of $474 \mathrm{~mm}$, providing a finite beam with phase curvature at the sample plane. The detector used was an Apogee Alta CCD (charge-coupled device) camera placed $0.59 \mathrm{~m}$ from the focus with $2,048 \times 2,048$ pixels, each square and $14 \mu \mathrm{m}$ across, that could be scanned in both the horizontal and vertical directions.

The optical data presented in this article comprised a total of 480 individual CCD frames: 400 short $(0.2 \mathrm{~s})$ exposures for the intense central region and 80 long $(4 \mathrm{~s})$ exposures to capture the higher-angle scatter. In order to record the entire diffraction pattern, the detector was mounted on two Newport stages, enabling it to be scanned in both transverse directions. The individual frames from both the central and high-angle-scatter regions were then 'stitched' together to give a total array size of $4,096 \times 4,096$ pixels (equal to twice the size of the CCD). The propagated ESW was reconstructed out to the edges of this array, giving a predicted resolution of $\Gamma=1.22 \lambda / \mathrm{NA}=8 \mu \mathrm{m}$ (where $\lambda$ is the wavelength of the incident beam and NA is the numerical aperture of the detector), consistent with the CDI reconstruction of the resolution target.

Image reconstruction was carried out using $\mathrm{ER}^{10}$. The $(k+1)$ th iterate using ER may be described by the following equation (see ref. 9 for a full description of the notation used):

$$
\rho_{k+1}=\pi_{\mathrm{s}} \pi_{\mathrm{m}} \rho_{k},
$$

where $\rho_{k}$ is the iterate on the $k$ th iteration, and $\pi_{\mathrm{s}}$ and $\pi_{\mathrm{m}}$ are operators representing the support and modulus constraints respectively. The estimate for the sample ESW is defined here as $\pi_{\mathrm{m}} \rho_{k}$. In essence, the reconstruction takes place in two parts: the first part involves the recovery of the complex incident illumination, which is described in ref. 18. This is a key step in the procedure, as a thorough knowledge of the incident wavefield is vital to use the illumination to define the support. The second part involves finding a solution for $\pi_{\mathrm{m}} \rho_{k}$ via a free-space propagation of the ESW between the sample plane and the detector plane. In the detector plane the complex illumination (recovered in the first part) is added and consistency with the measured intensity enforced. The illumination is then subtracted and the ESW is back-propagated to the sample plane, where the support constraint is applied. The whole process is then repeated until convergence of the algorithm is achieved.

For the visible-light reconstructions an initial guess for the support is made using a random set of phases with the shape determined by the illumination at the sample. At each iteration the support was then tightened by convolving the iterate with a one-pixel-variance gaussian function thresholded at approximately $10 \%$ of the maximum value of the image. The progress of the reconstruction was monitored using the error metric, defined in the detector plane as

$$
\chi^{2}=\frac{\sum_{n=0}^{N-1}\left[\left|\sqrt{I^{\mathrm{m}}(n)}\right|-\left|\left[\Im \rho_{k}\right](n)\right|\right]^{2}}{\sum_{n=0}^{N-1} I^{\mathrm{m}}(n)},
$$

where $I^{\mathrm{m}}(n)$ is the measured intensity in the $n$th pixel and $\Im$ is defined as the propagation operator. After just four iterations (equivalent to a few minutes computation time on a Pentium 4 desktop PC) the support was found to already resemble its final shape and could be used to generate an approximate solution for the sample ESW. To obtain the best result the iterative cycle was continued until a minimum value for the error metric was achieved, that is, the change in $\chi^{2}$ was less than $1 \times 10^{-5}$ between iterations. The error metrics for the four reconstructions in Fig. 2 (after 100 iterations) were all less than $\chi^{2}=4 \times 10^{-3}$.

The X-ray data were collected at beamline 2-ID-B of the Advanced Photon Source (APS). A Fresnel zone plate (FZP) with a nominal outer-zone width of $50 \mathrm{~nm}$ and a focal length of $14 \mathrm{~mm}$ at $2.17 \mathrm{keV}$ was used to generate the curved incident illumination. A $28 \mu \mathrm{m}$ central stop upstream of the FZP and a $20 \mu \mathrm{m}$ order-sorting aperture were also included in the X-ray set-up to remove the higher-order contributions from the FZP and the direct beam. A Peltier-cooled Princeton Instruments PIXIS CCD detector with 2,048 $\times 2,048$ pixels, each $13.5 \mu \mathrm{m}$, and placed $0.55 \mathrm{~m}$ from the focal plane, was used to record the intensity distribution of the diffracted $\mathrm{X}$-rays. The diffraction pattern from the sample extends to the edges of the $1,820 \times 1,820$ pixel array on all four sides (Fig. 3b), equivalent to a diffraction angle of $2.6^{\circ}$ at the mid-point of the edge of the array. The pixel size in the plane of the sample is therefore $12.9 \mathrm{~nm}$ and the predicted spatial resolution is $16 \mathrm{~nm}$.

The sample used for the soft-X-ray experiments was an X40-30-2 calibration target (Xradia, USA) positioned $450 \mu \mathrm{m}$ from the focus. The imaged 
region was a gold spoked star pattern (with height $=150 \pm 10 \mathrm{~nm}$ ) deposited onto a free-standing $\mathrm{Si}_{3} \mathrm{~N}_{4}$ membrane ( $\sim 110 \mathrm{~nm}$ thick). In the experimental set-up (described in ref. 19) the incident beam was kept in vacuo until the central stop. After traversing the sample the beam entered an evacuated flight path. The $160 \mu \mathrm{m}$ FZP was almost fully coherently illuminated in both transverse directions.

Analysis of X-ray data was identical to that of the optical experiments except that the shape of the support was kept fixed as a circle, the size of which was defined by the known extent of the illumination. As expected ${ }^{9}$, convergence of the algorithm is extremely rapid and a final solution for the reconstruction is typically reached in well under 100 iterations. In addition, the use of a central stop close to the FZP, rather than a beamstop in the vicinity of the sample plane means that we retain information from the lowest spatial frequencies in the diffraction pattern.

A total of 700 frames of data with a $2 \mathrm{~s}$ exposure time were collected from the spoked star resolution target; however, the method is very sensitive to drift between the sample and FZP. Hence, 36 frames of data (with a correlation between frames $>0.996$ ) were summed for the results presented in Figs 3,4. For the reconstruction of the complex ESW, 100 independent trials were carried out, each starting with a random set of phases and each completed with $\chi^{2}<1.3 \times 10^{-4}$. Between reconstructions there were only very minor variations in the sample ESW and the final image for each trial (arrived at after just 25 iterations) contained all of the features consistent with the specifications for the star pattern. The reconstruction of the sample in Fig. 4 is the result obtained from taking the average of 100 reconstructions. The final image shows the product of the amplitude and phase components of the complex ESW thresholded to show only the real part, which is consistent with the known information about the gold star pattern. For comparison, the phase of the complex ESW (without thresholding) is shown in Supplementary Information, Fig. S1.

Received 1 October 2007; accepted 31 January 2008; published 9 March 2008.

\section{References}

1. Chapman, H. N. et al. Femtosecond diffractive imaging with a soft-X-ray free-electron laser. Nature Phys, 2, 839-843 (2006).

2. Bates, R. H. T. Fourier phase problems are uniquely solvable in more than one dimension, 1. Underlying theory. Optik 61, 247-262 (1982)
3. Marchesini, S. et al. X-ray image reconstruction from a diffraction pattern alone. Phys. Rev. B 68 140101 (2003).

4. Miao, J., Charalambous, P., Kirz, J. \& Sayre, D. Extending the methodology of X-ray crystallography to allow imaging of micrometre-sized non-crystalline specimens. Nature $\mathbf{4 0 0}$, 342-344 (1999).

5. Robinson, I. K. et al. Reconstruction of the shapes of gold nanocrystals using coherent X-ray diffraction. Phys. Rev. Lett. 87, 195505 (2001).

6. Pfeifer, M. A. et al. Three-dimensional mapping of a deformation field inside a nanocrystal. Nature 442, 63-66 (2006).

7. Shapiro, D. et al. Biological imaging by soft $\mathrm{x}$-ray diffraction microscopy. Proc. Natl Acad. Sci. 102, 15343-15346 (2005)

8. Miao, J., Sayre, D. \& Chapman, H. N. Phase retrieval from the magnitude of the Fourier transforms of nonperiodic objects. J. Opt. Soc. Am. A 15, 1662-1669 (1998).

9. Williams, G. J. et al. Fresnel coherent diffractive imaging. Phys. Rev. Lett. 97, 025506 (2006).

10. Pitts, T. A. \& Greenleaf, J. F. Fresnel transform phase retrieval from magnitude. IEEE Trans. Ultrason. Ferroelectr. Freq. Control 50, 1035-1045 (2003).

11. Fienup, J. R. Phase retrieval algorithms: A comparison. Appl. Opt. 21, 2758-2769 (1982).

12. Gabor, D. Microscopy by reconstructed wavefronts. Proc. R. Soc. Lond. A 197, 454-487 (1949).

13. Walton, A. J. The Abbe theory of imaging: An alternative derivation of the resolution limit. Eur. J. Phys. 7, 62-63 (1986).

14. Williams, G. J., Quiney, H. M., Peele, A. G. \& Nugent, K. A. Coherent diffractive imaging and partial coherence. Phys. Rev. B 75, 104102 (2007).

15. Rodenburg, J. M. et al. Hard-X-ray lensless imaging of extended objects. Phys. Rev. Lett. 98 034801 (2007).

16. Spence, J. C. H., Weierstall, U. \& Howells, M. Coherence and sampling requirements for diffractive imaging. Ultramicroscopy 101, 149-152 (2004).

17. Zuo, J. M. et al. Atomic resolution imaging of a carbon nanotube from diffraction intensities. Science 300, 1419-1421 (2003).

18. Quiney, H. M. et al. Diffractive imaging of highly focused X-ray fields. Nature Phys. 2, 101-104 (2006).

19. McNulty, I. et al. A beamline for $1-4 \mathrm{keV}$ microscopy and coherence experiments at the Advanced Photon Source (APS). Rev. Sci. Instrum. 67, 3372 (1996).

\section{Acknowledgements}

We acknowledge L. Whitehead for assistance in acquiring optical data. We also acknowledge the support of the Australian Research Council Centre of Excellence for Coherent X-Ray Science and the Australian Synchrotron Research Program. Use of the Advanced Photon Source was supported by the US Department of Energy, Office of Science, Office of Basic Energy Sciences, under contract No. DE-AC02-06CH11357.

Correspondence and requests for materials should be addressed to G.J.W.

Supplementary Information accompanies this paper on www.nature.com/naturephysics.

Author contributions

B.A., G.J.W., J.N.C., M.A.P., M.D.J. and I.M. made the X-ray measurements. B.A. carried out visible light experiments. B.A. and G.J.W. were responsible for data analysis and project planning. K.A.N.

G.J.W. and A.G.P. were responsible for the initial concept. All authors contributed to the writing of the manuscript.

Reprints and permission information is available online at http://npg.nature.com/reprintsandpermissions/ 